\title{
Alterations in erythrocytes in hyperosmolar diabetic decompensation: a pathophysiological basis for impaired blood flow and for an improved design of fluid therapy
}

\author{
L.A. Evan-Wong ${ }^{1}$, R. J. L. Davidson ${ }^{1}$ and J. M. Stowers ${ }^{2}$ \\ ${ }^{1}$ Haematology Unit and ${ }^{2}$ Diabetic Department, University of Aberdeen, Scotland, UK
}

\begin{abstract}
Summary. In a study of 10 diabetic patients, each of whom was in a severely decompensated state, notable alteration of blood flow properties was observed in those six patients who were hyperosmolar. In this form of diabetic decompensation, whole blood filtration was distinctly impaired. The additional impairment was shown to be due to an accumulation of solute within the erythrocytes occurring as a consequence of hyperosmolarity. The alterations in erythrocytes were revealed by Coulter blood count abnormalities and confirmed by osmotic fragility studies. When biochemical improvement was achieved in these patients, rapid resolution of the erythrocyte abnormalities occurred. Microvascular ischaemia due to such
\end{abstract}

erythrocyte alterations may be a possible explanation for the characteristic cerebral disturbances of the hyperosmolar diabetic state. Altered blood flow properties would also promote vascular thrombosis, a common terminal event in the hyperosmolar non-ketotic syndrome with associated 50 per cent mortality. An improved design of the insulin and fluid replacement therapy for patients in hyperosmolar diabetic coma might be based on the findings of these and further studies.

Key words: Hyperosmolar diabetic coma, erythrocytes, diabetes.
Severe ketoacidosis and the hyperosmolar non-ketotic syndrome are both associated with significant mortality $(10 \%$ and $50 \%$ respectively) which may be related to impairment of blood flow causing terminal thrombotic events [1, 2 19]. The depressed conscious level characteristic of hyperosmolar diabetic decompensation and the occurrence of cerebral oedema during diabetic decompensation may both be related to impaired cerebral haemoperfusion and oxygen delivery $[3,4]$.

This study describes novel changes in the blood of diabetic patients with severe decompensation which have particular relevance to blood flow. Further studies of these abnormalities would clarify the pathophysiology of morbid events in hyperosmolar decompensation and could help in formulating a better approach to fluid replacement therapy for this particular category of patient. Currently, diverse views are held on the management of such patients, and their outcome is less than satisfactory [5-8].

\section{Subjects and methods}

\section{Patients}

Whenever practicable over a 13 -month period, any patient who was admitted to the various medical units in the Foresterhill group of hospitals in Aberdeen, Scotland with a diagnosis of diabetic ketoacidosis was ascertained for research study. Ten such patients were obtained and their subsequent management was according to the policy of the unit involved. Intravenous insulin infusion regimes were used by all units, but fluid replacement strategy varied widely.

The conscious level of each patient on admission was clinically graded as follows: Grade 1 - alert and responds coherently to questions, Grade 2 - drowsy but responds to minimal stimulation, Grade 3 - minimal response to maximal stimulation, Grade 4 - responds to deep pain only.

\section{Methods}

On admission and after biochemical recovery, the following investigations were routinely undertaken: peripheral blood profile (Coulter $S$ standardised with $4 C)$ on venous blood with $\mathrm{K}_{2} \operatorname{EDTA}(1 \mathrm{mg} / \mathrm{ml})$ as anticoagulant; microscopy of native blood film; microhaematocrit $1200 \mathrm{~g}$ for $3 \mathrm{~min}$ (Hawksley centrifuge, Gelman Instruments, London, UK); biochemical profile using a sequential multiple analyser with computer (Technicon Instruments, Tarrytown, New York, USA); serum osmolarity measured by depression of freezing point (Osmette A, Precision Systems, Sudbury, Mass, USA). Arterial puncture for blood gasses and $\mathrm{pH}$ (Model C 13, Instrumentation Labs, Dugnano, Italy) were done only when deemed necessary by the clinical management team.

Osmotic fragility studies were carried out on heparinised samples (Vacutainer, Becton-Dickinson, Rutherford, New Jersey, USA) by a standard method [9] and the results expressed as the mean corpuscular fragility (MCF) - (percentage $\mathrm{NaCl}$ at which $50 \%$ lysis occurs). Whole blood filterability was measured on fresh blood $\left(\mathrm{K}_{2}\right.$ EDTA,

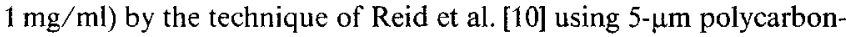
ate filters (Nucleopore Corporation, Pleasanton, California, USA). 
Table 1. Haematological and rheological data from the decompensated diabetic patients studied

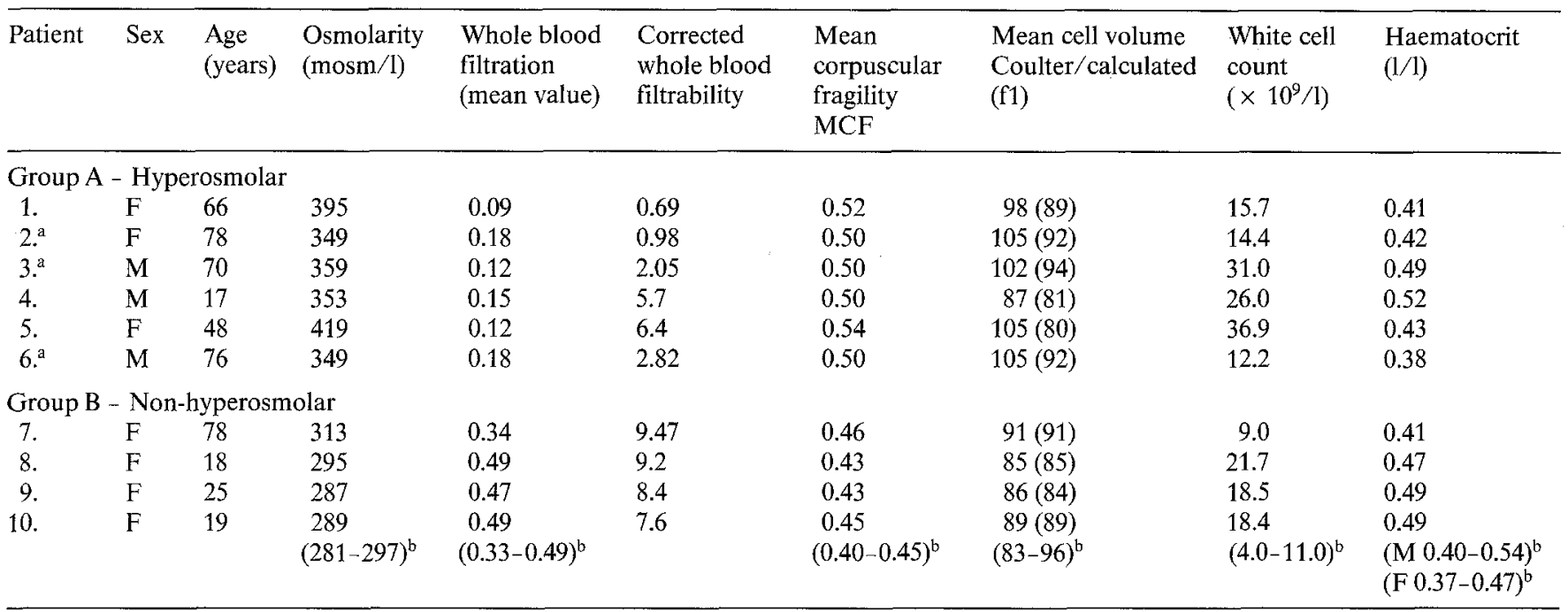

${ }^{a}$ Died during this admission; ${ }^{b}$ reference range for laboratory

Table 2. Biochemical data and conscious level grading of patients on admission

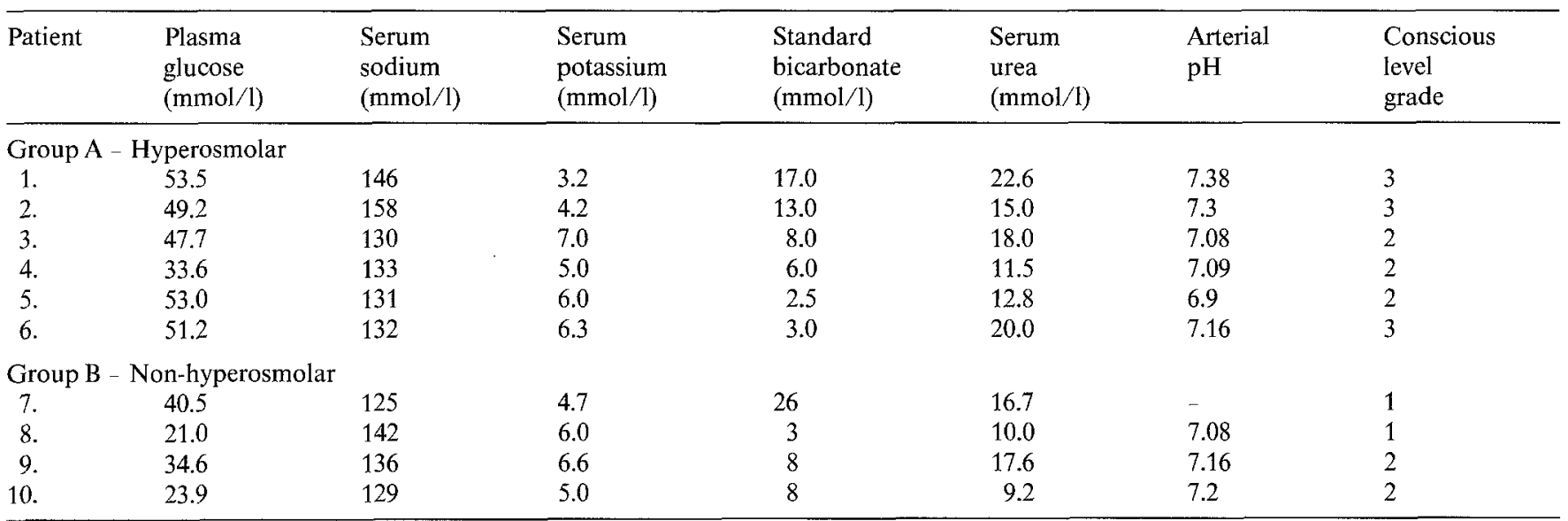

Tests were done on duplicate samples within 30 min of obtaining the blood and results are expressed as:

(a) whole blood filterability

$$
=\frac{60 \times \text { haematocrit }}{\text { filtration time in seconds for } 1 \mathrm{ml} \text { of blood }}
$$

where account is taken of the concentration of erythrocytes [10] and (b) as corrected whole blood filterability where filtration is further standardised for a white cell count of $7.5 \times 10^{9} / 1[11]$.

\section{Results}

The patients suffered from various types of diabetic decompensation and are divided into two categories, hyperosmolar (group A) and non-hyperosmolar (group B) as shown in Table 1. Three major abnormalities were evident in the group A patients. Whole blood filterability was notably impaired in comparison with the non-hyperosmolar group B patients, erythrocyte osmotic fragility was abnormal in group A and the MCV (mean erythrocyte cell volume) values obtained from the Coulter counter were elevated. The MCV derived from a di-

rectly centrifuged haematocrit $\left(\mathrm{MCV}=\frac{\text { haematocrit }}{\text { erythrocyte }}\right.$ count $)$

was, in fact, normal (Table 1).

The biochemical data of the patients and conscious level grading are shown in Table 2. In group A, patients 1-2 were hyperosmolar non-acidotic. Patients 3-6 were hyperosmolar and in addition were ketoacidotic. In contrast, the patients 7-10 in group B were not hyperosmolar but were ketotic. Cerebral impairment was more often manifest in group A. The impaired whole blood filterability, the elevated Coulter MCV measurements and the abnormal erythrocyte osmotic fragility observed in these same patients returned rapidly to normal following biochemical improvement. The accompanying osmotic fragility curves shown in Figure 1 demonstrate the typical pattern which occurred. 


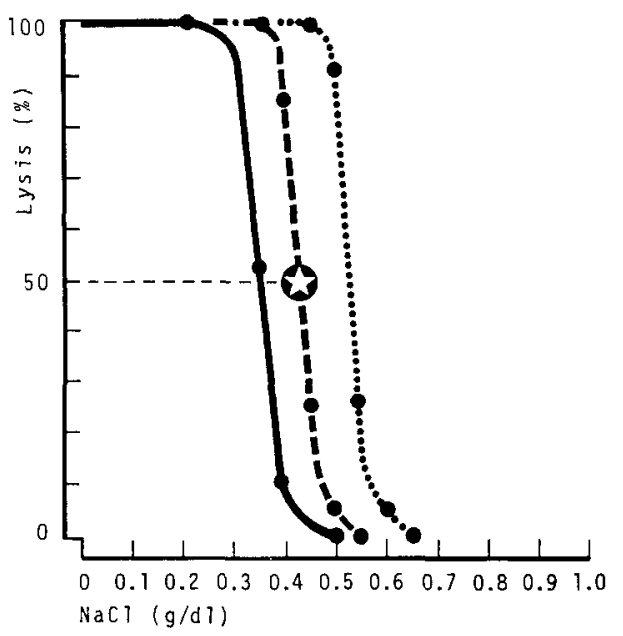

Fig. 1. Typically abnormal erythrocyte osmotic fragility found: a during hyperosmolar diabetic decompensation $\cdot \ldots . . . . \cdot \bullet$ and $\mathbf{b}$ after recovery $\bullet \longrightarrow$ (actually taken from patient 2 , Table 1 ). The normal control curve -.-.-. has also been drawn and the mean corpuscular fragility (MCF) is indicated by $\star$ where $50 \%$ lysis of normal erythrocytes occurs

\section{Discussion}

The marked impairment in red cell filterability encountered in the group A patients can be explained on the basis of the four variables which determine erythrocyte deformability, namely (a) the mechanical properties of the cell membrane, (b) the internal viscosity of the cell contents, (c) erythrocyte morphology and (d) the ratio of surface membrane area to volume of the cell [12]. In the severely decompensated diabetic patient who has also become hyperosmolar, each of these four variables related to erythrocyte deformability is unfavourably altered, producing a summated effect.

Firstly, using micropipette techniques and slow motion cinephotography, previous investigators have demonstrated abnormal erythrocyte membrane behaviour in diabetes $[13,14]$. Secondly, the internal viscosity of the cell content will be increased in these patients by virtue of hyperosmolarity and intracellular dehydration [15]. Thirdly, the erythrocytes in severely decompensated diabetic patients have been noted to be of abnormal morphology, and to assume echinocytic (finely crenated) shapes [16], possibly related to the effect of fatty acids [17]. Finally, we have fulfilled the fourth criterion for reduced erythrocyte deformability by demonstrating that the erythrocytes in hyperosmolar diabetic patients also have an abnormal volume to surface membrane area ratio which is due to an accumulation of cytoplasmic solute.

Volume-to-surface area dysproportion was illustrated by osmotic fragility studies. The osmotic resistance of freshly obtained erythrocytes reflects their ability to take up water without lysis when introduced into increasingly hypotonic solutions and this in turn dependends on their volume-to-surface membrane area ratio.
The explanation for the anomalous Coulter MCV results in the hyperosmolar group of patients is similarly based on the accumulation of intra-erythrocytic solute. Mammalian erythrocytes in vivo exist in osmotic equilibrium with their plasmatic environment. During diabetic decompensation, erythrocytes in the circulation of these hyperosmolar patients must therefore also acquire a hypertonic intracellular content in order to maintain osmotic equilibrium with the surrounding plasma. When such erythrocytes are subsequently introduced into relatively hypotonic diluent as occurs in the process of Coulter counting, water will enter the cell and induce swelling, thereby leading to an erroneously high Coulter MCV value in vitro [16]. Thus, it is noticeable in Group A patients that their Coulter MCV values were erroneously higher (an average of $12 \%$ ) than the calculated $\mathrm{MCV}$ values derived from the centrifuged haematocrit, whereas the Coulter MCV is normally expected to be about $3 \%$ lower than its calculated counterpart. Indeed, this anomalous finding is of some value in confirming the diagnosis of hyperosmolarity associated with diabetic decompensation. The Coulter counter is a commonly used instrument and in these circumstances provides a readily available indirect measurement of tissue tonicity compared to that of Coulter diluents (Isoton II. Coulter Electronics, Luton, England. Osmolarity $=339 \mathrm{mosmol} / \mathrm{l})$.

The accumulated intra-erythrocytic solute subsequently disappeared when biochemical improvement occurred as demonstrated by the return of normal osmotic resistance of the erythrocytes (Fig. 1).

It is understandable that erythrocytes with an increased ratio of intracellular content to surface membrane area would have abnormal flow properties. Whereas the normal laxity of the cell membrane allows an erythrocyte to assume unusual, even dumb-bell shapes in order to pass through a short channel of diameter smaller than its own [18], cells which have more content or less membrane will show reduced deformability in vitro and are less able to traverse microvascular passages in vivo. Microvascular ischaemia and impaired tissue oxygen delivery might therefore contribute significantly to the morbid events associated with hyperosmolar diabetic decompensation. Furthermore, the level of erythrocyte 2,3 DPG is known to be abnormally low in diabetic ketoacidosis [19]. The resultant anoxia might account for the depressed sensorium and other cerebral manifestations characteristic of hyperosmolar diabetic decompensation which remain incompletely explained. Reduced erythrocyte deformability would also have a contributory role in arterial thrombosis, a frequent occurrence in non-ketotic hyperosmolar coma [1], accounting for one-third of deaths in patients with diabetic ketoacidosis [2].

Further studies of a larger number of patients are required and might be directed towards the identification of the physical and chemical characteristics of the solute(s) accumulating within erythrocytes of diabetic pat- 
ients suffering from hyperosmolar decompensation. The ability of such solute(s) to traverse cell membranes would influence the strategy adopted in choosing intravenous fluids and in lowering the blood glucose in this particular category of patient.

The haematological stigma described in this study might alert the clinician to diabetic decompensation which has been complicated by significant hyperosmolarity and to its attendant morbidity and mortality. The cerebral catastrophies which occur so frequently in these patients are more likely to be due to the vascular complications of hyperosmolarity and not the result of treatment as has been stated in the past. Furthermore, early recognition of hyperosmolarity and use of hypotonic solutions would expedite normalisation of the perfusion characteristics of blood and help to restore the microvascular circulation.

Acknowledments. We are extremely grateful to A. Shepherd, who typed the script with skill and patience.

\section{References}

1. Clements RS, Vourganti B (1978) Fatal diabetic ketoacidosis: major causes and approaches to their prevention. Diabetes Care 1: 314-325

2. Whelton MJ, Walde D, Havard CWH (1971) Hyperosmolar nonketotic diabetic coma: with particular reference to vascular complications. Br Med J 1: 85-86

3. Gordon EE, Kabadi VM (1976) The hyperglycaemic hyperosmolar syndrome. Am J Med Sci 271: 253-268

4. Arieff A, Carroll HJ (1974) Cerebral oedema and depression of sensorium in non-ketotic hyperosmolar coma. Diabetes 23: $525-531$

5. Editorial (1982) Crystalloid infusions in diabetic ketoacidosis. Lancet 2: 308-309

6. Hillman KM (1982) Crystalloid infusions in diabetes. Lancet 2: 548 (Letter)

7. Axelrod L (1982) Crystalloid infusions in diabetes. Lancet 2: 549 (Letter)

8. Matz R, Carroll P (1982) Crystalloid infusions in diabetes. Lancet 2: 549 (Letter)
9. Dacie JV, Lewis SM, Gordon-Smith EC (1975) Laboratory methods used in the investigation of the haemolytic anaemias. II. Hereditary haemolytic anaemias. In: Dacie JV, Lewis SM (eds) Practical haematology, 5th ed. Churchill Livingston, Edinburgh, pp 202-208

10. Reid HL, Barnes AJ, Lock PJ, Dormandy JA, Dormandy JL (1976) A simple method for measuring erythrocyte deformability. Technical methods. J Clin Pathol 29: 855-857

11. Alderman MJ, Ridge A, Morley AA, Ryall RG, Walsh JA (1981) Effect of total leucocyte count on whole blood filterability in patients with peripheral vascular disease. J Clin Pathol 34: 163-166

12. Working Group Report on Red Cell Deformability (1981) Second European conference on clinical haemorheology London September 1981 Russell Wilkes Associates, London, UK, pp 1-8

13. McMillan DE, Utterback NG, La Puma J (1978) Reduced erythrocyte deformability in diabetes. Diabetes 27:895-901

14. Williamson JR, Kilo C, Sutera SP, Gardner RA, Boylan CW, Chang KC (1981) Shear induced deformation of red cells: decreased elongation and membrane rotation in diabetes mellitus. Horm Metab Res [Suppl] 11: 103-104

15. Schmid-Schonbein H, Volger E (1976) Red-cell aggregation and red-cell deformability in diabetes. Diabetes 25 (Suppl) 2: 897-902

16. Evan-Wong LA, Davidson RF (1983) The abnormally elevated Coulter MCV in diabetic ketoacidosis, and its underlying association with marked plasma hyperosmolarity. J Clin Pathol 36: 334-336

17. Furchgott RF (1940) Disk-sphere transformation in mammalian red cells. J Exp Biol 17: 30-33

18. Schmid-Schonbein H (1970) In: The spleen. Lennert K, Haims D (eds) Springer, Berlin, pp 67-68

19. Ditzel $J$ (1970) Changes in rheology and oxygen transport functions of the erythrocytes in diabetes. In: Ditzel J, Lewis DH (eds) Microcirculatory approaches to current therapeutic problems. Karger, Basle, pp 123-131

Received: 10 September 1984

and in revised form: 14 May 1985

Dr. L.A. Evan-Wong

Department of Haematology

Dunfermline \& West Fife Hospital

Reid Street

Dunfermline

Fife

Scotland KY12 7EZ

UK 